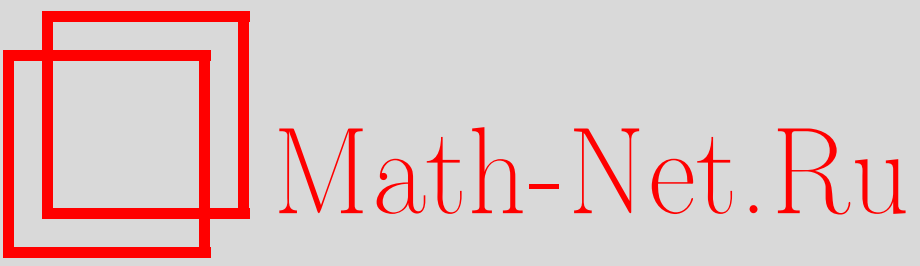

М. Нешпорски, Лестничная диаграмма Лапласа дискретных уравнений лапласова типа, ТМФ, 2002, том 133, номер 2, 301-310

DOI: https://doi.org/10.4213/tmf399

Использование Общероссийского математического портала Math-Net.Ru подразумевает, что вы прочитали и согласны с пользовательским соглашением

http://www . mathnet.ru/rus/agreement

Параметры загрузки:

IP: 54.197 .130 .99

26 апреля 2023 г., 13:23:40 


\section{ЛЕСТНИЧНАЯ ДИАГРАММА ЛАПЛАСА ДИСКРЕТНЫХ УРАВНЕНИЙ ЛАПЛАСОВА ТИПА}

Вводится понятие лестничной диаграммы Лапласа для дискретных аналогов уравнений лапласова типа. Получены уравнение, сопряженное дискретному уравнению Мутара, и дискретный аналог нелинейного представления уравнения Гурса.

Ключевые слова: лестничная диаграмма Лапласа, решетка Тоды, дискретные иерархии КП.

\section{1. ОБОЗНАЧЕНИЯ}

Через $f(u, v)$ мы будем обозначать функции от непрерывных переменных, т.е. $f: \mathbb{R}^{2} \ni$ $(u, v) \mapsto f(u, v) \in \mathbb{R}$, а через $f\left(m_{1}, m_{2}\right)$ - функции от дискретных переменных, т.е. $f$ : $\mathbb{Z}^{2} \ni\left(m_{1}, m_{2}\right) \mapsto f\left(m_{1}, m_{2}\right) \in \mathbb{R}$. Частные производные обозначаются запятой, например $f_{\text {, } u v}(u, v):=\partial^{2} f(u, v) / \partial u \partial v$, а нижние индексы в скобках обозначают операторы сдвига, например $f\left(m_{1}, m_{2}\right)_{(1)}:=f\left(m_{1}+1, m_{2}\right), f\left(m_{1}, m_{2}\right)_{(2)}:=f\left(m_{1}, m_{2}+1\right)$, $f\left(m_{1}, m_{2}\right)_{(12)}:=f\left(m_{1}+1, m_{2}+1\right), f\left(m_{1}, m_{2}\right)_{(-1)}:=f\left(m_{1}-1, m_{2}\right)$ и т.д. Излишние аргументы функций опускаются в случае, когда сами операторы однозначно свидетельствуют о том, функции от каких - дискретных или непрерывных - переменных обсуждаются в данном случае. Разностные операторы обозначаются через $\Delta_{i} f:=f_{(i)}-f$. Введем также специальный оператор четверного отношения $\diamond f:=f_{(12)} f /\left(f_{(1)} f_{(2)}\right)$.

\section{2. ВВЕДЕНИЕ}

Уравнение Лапласа

$$
L \psi(u, v)=0,
$$

где $L$ представляет собой дифференциальный оператор

$$
L=\frac{\partial^{2}}{\partial u \partial v}-A(u, v) \frac{\partial}{\partial u}-B(u, v) \frac{\partial}{\partial v}-C(u, v),
$$

часто называется мастер-уравнением в теории $S$-интегрируемых систем. Эта точка зрения подкрепляется сушествованием широкого класса преобразований типа Дарбу, введенных Йонасом [1] и Эйзенхартом [2] (эти преобразования называются фундаментальными преобразованиями многокомпонентных иерархий уравнения Кадомцева-Петвиашвили), которые действуют на пространстве решений системы уравнений лапласова

*Instytut Fizyki Teoretycznej, Uniwersytet w Białymstoku, Białystok, Poland. E-mail: maciejun@fuw.edu.pl, maciejun@alpha.uwb.edu.pl 
типа и порождают преобразования Дарбу-Беклунда для уравнений Дарбу, равно как и формулировкой современного метода $\bar{\partial}$-одевания, примененного к уравнениям Дарбу Захаровым и Манаковым [3].

Как метод $\bar{\partial}$-одевания, так и более важная в данном контексте дискретная версия фундаментального преобразования, действующая на пространствах решений дискретных аналогов уравнений Лапласа,

$$
\begin{gathered}
\mathcal{L} \psi\left(m_{1}, m_{2}\right)=0, \\
\mathcal{L}=\Delta_{(1)} \Delta_{(2)}-\mathcal{A}\left(m_{1}, m_{2}\right) \Delta_{(1)}-\mathcal{B}\left(m_{1}, m_{2}\right) \Delta_{(2)}-\mathcal{C}\left(m_{1}, m_{2}\right),
\end{gathered}
$$

были применены к дискретным аналогам уравнений Дарбу [4], [5]. Теория редукций дискретного фундаментального преобразования была развита в работах [6]-[9].

С другой стороны, Аторн заметил [10], что симметрия лестничной диаграммы Лапласа уравнения лапласова типа накладывает ограничения на операторы $L$, входящие в уравнения лестничной диаграммы. В частности, исследовался случай, когда лестничная диаграмма Лапласа содержит уравнение Мутара

$$
\psi, u v=f(u, v) \psi
$$

и уравнение Гурса

$$
\psi, u v=\frac{1}{2}(\ln \lambda(u, v)),{ }_{v} \psi,{ }_{u}+\lambda(u, v) \psi
$$

которое, в свою очередь, связано с уравнением (детали соответствия см., например, в работах [11])

$$
\vartheta_{, x y}=2 \sqrt{\lambda(x, y) \vartheta_{, x} \vartheta_{, y}} .
$$

Преобразования типа Дарбу, которые сохраняют вид уравнения Мутара (5) или уравнения Гурса (6), были найдены соответственно в работах [12] и [11].

Опишем сначала основные моменты теории дискретных уравнений лапласова типа (инвариантное описание и $T$-эквивалентность вводятся в разделе 3 ). Затем определим две операции, действующие на инвариантах дискретного уравнения лапласова типа, а именно преобразования Лапласа в разделе 4 (следуя работе [13], мы вводим понятие цепочки Лапласа дискретных уравнений лапласова типа) и операцию сопряжения в разделе 5. Объединяя понятия цепочки преобразований Лапласа и операции сопряжения, в разделе 6 мы введем понятие лестничной диаграммы Лапласа дискретных уравнений лапласова типа. Лестничная диаграмма Лапласа оказывается полезной для описания как старых, так и новых вариантов дискретных уравнений Мутара, которые были введены соответственно в работах [6] и [14] (см. раздел 7), равно как и для описания дискретного уравнения Гурса [8] (см. раздел 8).

\section{3. ДИСКРЕТНОЕ УРАВНЕНИЕ ЛАПЛАСОВА ТИПА. Т-ЭКВИВАЛЕНТНОСТЬ}

Дифференциальный оператор (2) сохраняет вид при преобразовании

$$
L \mapsto \tilde{L}=\frac{1}{g} \circ L \circ g,
$$


где через $g$ обозначен оператор умножения на скалярную функцию $g=g(u, v) \neq 0$. Это преобразование обычно называется калибровочнылм преобразованием. Будем называть два уравнения Лапласа $L \psi(u, v)=0$ и $\tilde{L} \psi(u, v)=0$ әквивалентными тогда и только тогда, когда существует функция $g$ такая, что операторы $\tilde{L}$ и $L$ связаны преобразованием (8). Следующие функции (инварианты Лапласа) оказываются инвариантными относительно калибровочного преобразования:

$$
h=A B-A,{ }_{u}+C, \quad k=A B-B, v+C .
$$

Если инварианты операторов $L$ и $\tilde{L}$ совпадают $(k=\tilde{k}$ и $h=\tilde{h})$, то сушествует калибровочное преобразование, которое переводит $\tilde{L}$ в $L$. Упорядоченные пары инвариантов $(h, k)$ нумеруют классы эквивалентности уравнения $(1)$. Более подробное описание непрерывного случая содержится в работе Аторна [10].

Дискретное уравнение лапласова типа (3) можно записать в виде

$$
\psi_{(12)}-\alpha \psi_{(1)}-\beta \psi_{(2)}-\gamma \psi=0
$$

где

$$
\alpha:=\mathcal{A}+1, \quad \beta:=\mathcal{B}+1, \quad \gamma:=\mathcal{C}-\mathcal{A}-\mathcal{B}-1
$$

Как и в непрерывном случае, можно ввести комбинации

$$
\kappa:=\frac{\beta \alpha_{(2)}}{\gamma_{(2)}}, \quad n:=\frac{\alpha \beta_{(1)}}{\gamma_{(1)}},
$$

которые инвариантны относительно дискретных калибровочных преобразований $\mathcal{L} \mapsto$ $\mathcal{L} g / g_{(12)}$, являющихся дискретными аналогами калибровочного преобразования (8), в то время как $g$ обозначает оператор умножения на функцию $g=g\left(m_{1}, m_{2}\right) \neq 0$. Будем называть инварианты (12) основными инвариантами, поскольку любые два оператора $\mathcal{L}$ и $\tilde{\mathcal{L}}$, имеющие совпадаюшие инварианты $(n, \kappa)=(\tilde{n}, \tilde{\kappa})$, могут быть связаны калибровочным преобразованием.

В разделе 7 будут также введены порожденные инварианты, которые не обладают этим основным свойством. Вторичнъе инварианты первого типа имеют вид

$$
\mathcal{K}:=\kappa \kappa_{(1)}, \quad \mathcal{H}:=n n_{(2)},
$$

в то время как вторичные инварианты второго типа представляются в виде

$$
\mathbf{K}:=\frac{n_{(2)}}{\kappa}, \quad \mathbf{H}:=\frac{\kappa_{(1)}}{n} .
$$

Пары $(n, \kappa)$ нумеруют классы эквивалентности дискретных уравнений лапласова типа.

Если базисные инварианты двух дискретных операторов Лапласа $\mathcal{L}$ и $\tilde{\mathcal{L}}$ удовлетворяют соотношениям

$$
\kappa=T \tilde{\kappa}, \quad n=T \tilde{n},
$$

где $T$ - оператор сдвига, т.е. $T f\left(m_{1}, m_{2}\right)=f\left(m_{1}+k, m_{2}+l\right), k, l \in \mathbb{N}$, то такие уравнения называются $T$-эквивалентными, а соответствуюшие расширенные классы эквивалентности находятся в однозначном соответствии с $T(n, \kappa)$. В дальнейшем рассмотрении $T$-эквивалентность оказывается весьма существенной. 


\section{4. ПРЕОБРАЗОВАНИЯ ЛАПЛАСА И ЦЕПОЧКА ЛАПЛАСА}

Чтобы решить дискретное уравнение лапласова типа (3), попробуем факторизовать дискретный оператор Лапласа (4), что может быть сделано только лишь двумя способами:

$$
\begin{aligned}
\left(\Delta_{(1)}-\mathcal{B}\right)\left(\Delta_{(2)}-\mathcal{A}_{(-1)}\right) \psi-\mathfrak{h} \psi & =0, \\
\left(\Delta_{(2)}-\mathcal{A}\right)\left(\Delta_{(1)}-\mathcal{B}_{(-2)}\right) \psi-\mathfrak{k} \psi & =0,
\end{aligned}
$$

где

$$
\begin{aligned}
\mathfrak{h} & =\mathcal{C}-\mathcal{A}+\mathcal{A}_{(-1)}(\mathcal{B}+1)=\gamma+\alpha_{(-1)} \beta, \\
\mathfrak{k} & =\mathcal{C}-\mathcal{B}+\mathcal{B}_{(-2)}(\mathcal{A}+1)=\gamma+\beta_{(-2)} \alpha .
\end{aligned}
$$

Если одна из двух функций $\mathfrak{h}$ или $\mathfrak{k}$ тождественно равна нулю, то факторизация возможна (а уравнения при этом разрешимы в квадратурах). Если это не так, то можно ввести функции

$$
\begin{aligned}
& \psi^{\uparrow}:=\left(\Delta_{(2)}-\mathcal{A}_{(-1)}\right) \psi, \\
& \psi^{\downarrow}:=\left(\Delta_{(1)}-\mathcal{B}_{(-2)}\right) \psi,
\end{aligned}
$$

в терминах которых уравнения (15) и (16) принимают вид

$$
\begin{aligned}
& \psi=\frac{1}{\mathfrak{h}}\left(\Delta_{(1)} \psi^{\uparrow}-\mathcal{B} \psi^{\uparrow}\right), \\
& \psi=\frac{1}{\mathfrak{k}}\left(\Delta_{(2)} \psi^{\downarrow}-\mathcal{A} \psi^{\downarrow}\right) .
\end{aligned}
$$

Исключая функцию $\psi$ из уравнений (18), (19) с помошью формул (20), (21), можно получить, что функции $\psi^{\uparrow}$ и $\psi^{\downarrow}$ удовлетворяют уравнениям

$$
\begin{aligned}
& \psi_{(12)}^{\uparrow}=\frac{\mathfrak{h}_{(2)}}{\mathfrak{h}} \alpha_{(-1)} \psi_{(1)}^{\uparrow}+\beta_{(2)} \psi_{(2)}^{\uparrow}+\frac{\mathfrak{h}_{(2)}}{\mathfrak{h}} \gamma \psi^{\uparrow}, \\
& \psi_{(12)}^{\downarrow}=\alpha_{(1)} \psi_{(1)}^{\downarrow}+\frac{\mathfrak{k}_{(1)}}{\mathfrak{k}} \beta_{(-2)} \psi_{(2)}^{\downarrow}+\frac{\mathfrak{k}_{(1)}}{\mathfrak{k}} \gamma \psi^{\downarrow},
\end{aligned}
$$

а инварианты этих уравнений связаны с инвариантами уравнения (10):

$$
\begin{gathered}
n^{\uparrow}=\frac{n_{(-1)} n_{(2)}}{\kappa} \diamond \frac{1}{(1+n)_{(-1)}}, \quad \kappa_{(1)}^{\uparrow}=n_{(2)}, \\
n_{(2)}^{\downarrow}=\kappa_{(1)}, \quad \kappa^{\downarrow}=\frac{\kappa_{(-2)} \kappa_{(1)}}{n} \diamond \frac{1}{(1+\kappa)_{(-2)}}, \\
\mathbf{H}^{\uparrow}=(\mathbf{H} \diamond(1+n))_{(-1)}, \quad \mathbf{K}_{(1)}^{\uparrow}=\left(\mathbf{K}_{(1)} \diamond \frac{1}{1+n}\right)_{(2)}, \\
\mathbf{H}_{(2)}^{\downarrow}=\left(\mathbf{H}_{(2)} \diamond \frac{1}{1+\kappa}\right)_{(1)}, \quad \mathbf{K}^{\downarrow}=(\mathbf{K} \diamond(1+\kappa))_{(-2)} .
\end{gathered}
$$

Можно легко показать, что

$$
\left(n^{\uparrow \downarrow}, \kappa^{\uparrow \downarrow}\right)=\left(n^{\downarrow \uparrow}, \kappa^{\downarrow \uparrow}\right)=(n, \kappa),
$$


и преобразования Лапласа для дискретных уравнений лапласова типа, т.е. отображения $(n, \kappa) \mapsto\left(n^{\uparrow}, \kappa^{\uparrow}\right)$ и $(n, \kappa) \mapsto\left(n^{\downarrow}, \kappa^{\downarrow}\right)$, оказываются тем самым взаимно обратными. По аналогии с непрерывным случаем будем называть цепочку уравнений

$$
\ldots,\left(n^{\downarrow \downarrow}, \kappa^{\downarrow \downarrow}\right),\left(n^{\downarrow}, \kappa^{\downarrow}\right),(n, \kappa),\left(n^{\uparrow}, \kappa^{\uparrow}\right),\left(n^{\uparrow \uparrow}, \kappa^{\uparrow \uparrow}\right), \ldots
$$

цепочкой Лапласа дискретных уравнений лапласова типа. Сделаем три важных замечания. Во-первых, преобразования Лапласа хорошо определены на $T$-эквивалентных классах. Во-вторых, уравнение

$$
n_{(1)}^{\uparrow} n_{(2)}^{\downarrow}=n n_{(12)} \diamond \frac{1}{1+n},
$$

где преобразования Лапласа $\uparrow$ и $\downarrow$ рассматриваются как соответствующие сдвиги в положительном и отрицательном направлениях по третьей дискретной переменной, представляет собой одну из возможных форм записи уравнения Хироты [15]. Наконец, втретьих, вводя $\mathcal{X}:=\psi^{\uparrow} / b$ и $\mathcal{Y}:=\psi / a$, где $a$ и $b$ суть решения уравнений $b_{(1)}=\beta b$ и $a_{(2)}=\alpha_{(-1)} a$, можно представить уравнения (18) и (20) в виде

$$
\Delta_{(2)} \mathcal{Y}=\mathcal{Q X}, \quad \Delta_{(1)} \mathcal{X}=\mathcal{P} \mathcal{Y}
$$

где

$$
\mathcal{P}=\frac{a \mathfrak{h}}{b_{(1)}}, \quad \mathcal{Q}=\frac{b}{a_{(2)}},
$$

в то время как уравнения (22) и (10) в этой новой калибровке примут вид

$$
\begin{aligned}
& \mathcal{Y}_{(12)}=\mathcal{Y}_{(1)}+\frac{\mathcal{Q}_{(1)}}{\mathcal{Q}} \mathcal{Y}_{(2)}-\mathcal{M} \frac{\mathcal{Q}_{(1)}}{\mathcal{Q}} \mathcal{Y}, \\
& \mathcal{X}_{(12)}=\frac{\mathcal{P}_{(2)}}{\mathcal{P}} \mathcal{X}_{(1)}+\mathcal{X}_{(2)}-\mathcal{M} \frac{\mathcal{P}_{(2)}}{\mathcal{P}} \mathcal{X}
\end{aligned}
$$

где $\mathcal{M}=1-\mathcal{P} \mathcal{Q}$.

\section{5. ФУНДАМЕНТАЛЬНОЕ ПРЕОБРАЗОВАНИЕ И СОПРЯЖЕННЫЕ УРАВНЕНИЯ}

Фундаментальное преобразование, которое связывает пространства решений уравнения (1) с различными (но связанными) коэффициентными функциями, параметризуется двумя функциональными параметрами [2]. Первый из этих параметров есть решение уравнения (1), а второй функциональный параметр представляет собой решение уравнения, сопряженного уравнению (1):

$$
\psi^{\dagger},{ }_{u v}+\left(A \psi^{\dagger}\right){ }_{, u}+\left(B \psi^{\dagger}\right),{ }_{v}-C \psi^{\dagger}=0 .
$$

Введем теперь уравнение, сопряженное дискретному уравнению (10). Для этого необходимо найти фундаментальное преобразование [5] для дискретного уравнения лапласова типа (1).

6 Теоретическая и математическая физика, т. 133, № 2,2002 г. 
Дискретное фундаментальное преобразование отображает пространство решений уравнения (здесь удобно использовать так называемую аффинную калибровку $\mathcal{C}=0$ )

$$
\Delta_{(1)} \Delta_{(2)} x=\frac{\Delta_{(2)} a}{a} \Delta_{(1)} x+\frac{\Delta_{(1)} b}{b} \Delta_{(2)} x
$$

в пространство решений уравнения

$$
\Delta_{(1)} \Delta_{(2)} x^{1}=\frac{\Delta_{(2)} a^{1}}{a^{1}} \Delta_{(1)} x^{1}+\frac{\Delta_{(1)} b^{1}}{b^{1}} \Delta_{(2)} x^{1}
$$

Это преобразование имеет вид

$$
\begin{aligned}
& \frac{1}{\Delta_{(1)}\left(\frac{\vartheta^{\prime}}{\vartheta}\right)} \Delta_{(1)}\left(\frac{x^{1} \vartheta^{\prime}}{\vartheta}\right)=\frac{1}{\Delta_{(1)}\left(\frac{1}{\vartheta}\right)} \Delta_{(1)}\left(\frac{x}{\vartheta}\right), \\
& \frac{1}{\Delta_{(2)}\left(\frac{\vartheta^{\prime}}{\vartheta}\right)} \Delta_{(2)}\left(\frac{x^{1} \vartheta^{\prime}}{\vartheta}\right)=\frac{1}{\Delta_{(2)}\left(\frac{1}{\vartheta}\right)} \Delta_{(2)}\left(\frac{x}{\vartheta}\right) .
\end{aligned}
$$

Функциональные параметры $\vartheta$ и $\vartheta^{\prime}$ этого преобразования оказываются не произвольными: функция $\vartheta$ должна быть решением уравнения (32), т.е.

$$
\Delta_{(1)} \Delta_{(2)} \vartheta=\frac{\Delta_{(2)} a}{a} \Delta_{(1)} \vartheta+\frac{\Delta_{(1)} b}{b} \Delta_{(2)} \vartheta
$$

а функция $\vartheta^{\prime}$ должна строиться из $\vartheta$ и решения $\vartheta^{\dagger}$ уравнения

$$
\Delta_{(1)} \Delta_{(2)} \vartheta^{\dagger}+\Delta_{(1)}\left(\frac{\frac{\Delta_{(2)} a}{a} \vartheta^{\dagger}}{1+\frac{\Delta_{(2)} a}{a}+\frac{\Delta_{(1)} b}{b}}\right)+\Delta_{(2)}\left(\frac{\frac{\Delta_{(1)} b}{b} \vartheta^{\dagger}}{1+\frac{\Delta_{(2)} a}{a}+\frac{\Delta_{(1)} b}{b}}\right)=0 .
$$

Чтобы найти $\vartheta^{\prime}$, надо сначала найти вспомогательные функции $\lambda$ и $\chi$ такие, что

$$
\begin{gathered}
\Delta_{(1)} \lambda=\frac{\frac{\Delta_{(1)} b}{b} \vartheta^{\dagger}}{1+\frac{\Delta_{(2)} a}{a}+\frac{\Delta_{(1)} b}{b},} \quad \Delta_{(2)} \lambda=-\Delta_{(2)} \vartheta^{\dagger}-\frac{\frac{\Delta_{(2)} a}{a} \vartheta^{\dagger}}{1+\frac{\Delta_{(2)} a}{a}+\frac{\Delta_{(1)} b}{b}}, \\
\chi=\lambda+\vartheta^{\dagger} .
\end{gathered}
$$

Тогда искомая функция $\vartheta^{\prime}$ удовлетворяет уравнениям

$$
\Delta_{(1)} \vartheta^{\prime}=\chi \Delta_{(1)} \vartheta, \quad \Delta_{(2)} \vartheta^{\prime}=\lambda \Delta_{(2)} \vartheta .
$$

Условия (35)-(38) обеспечивают то, что функция $x^{1}$, задаваемая условиями (34), удовлетворяет уравнению (33). Новые функции $a^{1}$ и $b^{1}$ связаны с исходными функциями $a$ и $b$ следуюшим образом:

$$
\frac{\Delta_{(2)} a^{1}}{a^{1}}=\frac{\Delta_{(2)}\left(a \chi \frac{\vartheta}{\vartheta^{\prime}}-a\right)}{\left(a \chi \frac{\vartheta}{\vartheta^{\prime}}-a\right)}, \quad \frac{\Delta_{(1)} b^{1}}{b^{1}}=\frac{\Delta_{(1)}\left(b \lambda \frac{\vartheta}{\vartheta^{\prime}}-b\right)}{\left(b \lambda \frac{\vartheta}{\vartheta^{\prime}}-b\right)} .
$$

Будем называть уравнение (36) сопряженным уравнению (35). Инварианты $n^{\dagger}, \kappa^{\dagger}, \mathbf{H}^{\dagger}$ и $\mathbf{K}^{\dagger}$ уравнения (36) связаны с инвариантами $n, \kappa, \mathbf{H}$ и $\mathbf{K}$ уравнения (35):

$$
\begin{aligned}
& n^{\dagger}=\kappa_{(1)}, \quad \kappa^{\dagger}=n_{(2)}, \\
& \mathbf{H}^{\dagger}=\mathbf{K}_{(1)}, \quad \mathbf{K}^{\dagger}=\mathbf{H}_{(2)} .
\end{aligned}
$$

Будем называть уравнение $\mathcal{L}^{\dagger} \psi^{\dagger}=0$ сопряженным уравнению $\mathcal{L} \psi=0$ тогда и только тогда, когда

$$
T\left(n^{\dagger}, \kappa^{\dagger}\right)=T\left(\kappa_{(1)}, n_{(2)}\right) .
$$

Заметим, что $T(n, \kappa)^{\dagger \dagger}=T(n, \kappa)$. 


\section{6. ЛЕСТНИЧНАЯ ДИАГРАММА ЛАПЛАСА}

Рассматривая суперпозиции преобразований Лапласа и сопряжения, получим

$$
\begin{aligned}
& \left(n^{\uparrow \dagger}, \kappa^{\uparrow \dagger}\right)=\left(\frac{n_{(-1)} n_{(2)}}{\kappa} \diamond \frac{1}{1+n_{(-1)}}, n\right)_{(2)}, \\
& \left(n^{\dagger \downarrow}, \kappa^{\dagger \downarrow}\right)=\left(\frac{n_{(-1)} n_{(2)}}{\kappa} \diamond \frac{1}{1+n_{(-1)}}, n\right)_{(1)}, \\
& \left(n^{\downarrow \dagger}, \kappa^{\downarrow \dagger}\right)=\left(\frac{\kappa_{(-2)} \kappa_{(1)}}{n} \diamond \frac{1}{1+\kappa_{(-2)}}, \kappa\right)_{(1)}, \\
& \left(n^{\dagger \uparrow}, \kappa^{\dagger \uparrow}\right)=\left(\frac{\kappa_{(-2)} \kappa_{(1)}}{n} \diamond \frac{1}{1+\kappa_{(-2)}}, \kappa\right)_{(2)} .
\end{aligned}
$$

Таким образом, имеем

$$
T\left(n^{\uparrow \dagger}, \kappa^{\uparrow \dagger}\right)=T\left(n^{\dagger \downarrow}, \kappa^{\dagger \downarrow}\right), \quad T\left(n^{\downarrow \dagger}, \kappa^{\downarrow \dagger}\right)=T\left(n^{\dagger \uparrow}, \kappa^{\dagger \uparrow}\right),
$$

в результате получается коммутативная диаграмма, называемая лестничной диаграммой Лапласа дискретньх уравнений лапласова типа:

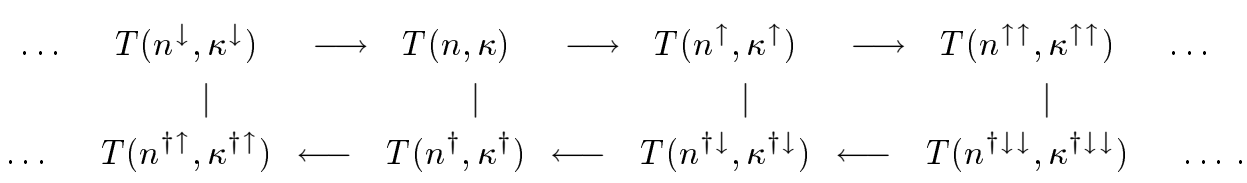

В этой диаграмме стрелки обозначают преобразования Лапласа $\uparrow$, действующие в “положительном" направлении, в то время как вертикальные линии ("перекладины лестнищы" ) обозначают операции сопряжения $\dagger$.

\section{7. ПОДКЛАСС ПРЕОБРАЗОВАНИЙ МУТАРА}

Уравнение Лапласа называется дискретным уравнением Мутара тогда и только тогда, когда его инварианты удовлетворяют соотношению $\mathbf{H}=\mathbf{K}$, или, что эквивалентно, $\mathcal{H}=\mathcal{K}$ или $n_{(2)} n=\kappa_{(1)} \kappa$. Каждое дискретное уравнение Мутара может быть преобразовано к виду

$$
N_{(12)}+N=F\left(N_{(1)}+N_{(2)}\right)
$$

с помошью калибровочного преобразования. Уравнение (45) вместе с преобразованием типа Дарбу было введено Ниммо и Шифом [6].

Из формул (41) следует, что естественным инвариантом уравнения, сопряженного дискретному уравнению Мутара, является величина

$$
\mathbf{H}_{(2)}^{\dagger}=\mathbf{K}_{(1)}^{\dagger}
$$

Таким образом, в отличие от непрерывного случая, дискретное уравнение Мутара оказывается несамосопряженным. Уравнение типа Лапласа называется сопряжсенным дискретному уравнению Мутара тогда и только тогда, когда его инварианты связаны 
преобразованием (46). Всякое сопряженное дискретное уравнение Мутара приводится с помощью калибровочного преобразования к уравнению

$$
\mathcal{N}_{(12)}+\mathcal{N}=F_{(1)} \mathcal{N}_{(1)}+F_{(2)} \mathcal{N}_{(2)}
$$

В случае, когда функция $N$ удовлетворяет дискретному уравнению Мутара (45), функция $\mathcal{N}$ вида

$$
\mathcal{N}:=N_{(1)}+N_{(2)}
$$

удовлетворяет сопряженному дискретному уравнению Мутара (47). В силу соотношения (48) уравнение (47) "наследует" свойства интегрируемости, присущие уравнению (45). В частности, дискретное преобразование Мутара [6] приводит к условиям

$$
\begin{aligned}
& \left(\mathcal{N}^{\prime} \frac{\Theta_{(1)}}{\Theta}+\mathcal{N}\right)_{(1)}=\frac{\left(\Theta_{(1)}+\Theta_{(2)}\right)_{(1)}}{\Theta_{(1)}+\Theta_{(2)}}\left(\mathcal{N}^{\prime} \frac{\Theta_{(2)}}{\Theta_{(12)}}+\mathcal{N}\right), \\
& \left(\mathcal{N}^{\prime} \frac{\Theta_{(2)}}{\Theta}-\mathcal{N}\right)_{(2)}=\frac{\left(\Theta_{(1)}+\Theta_{(2)}\right)_{(2)}}{\Theta_{(1)}+\Theta_{(2)}}\left(\mathcal{N}^{\prime} \frac{\Theta_{(1)}}{\Theta_{(12)}}-\mathcal{N}\right),
\end{aligned}
$$

где $\Theta$ есть решение дискретного уравнения Мутара (45):

$$
\Theta_{(12)}+\Theta=F\left(\Theta_{(1)}+\Theta_{(2)}\right) \text {. }
$$

Свойства уравнения (47) детально обсуждаются в работе [16].

\section{8. СИММЕТРИЧЕСКИЙ ПОДКЛАСС РЕШЕНИЙ УРАВНЕНИЙ ТИПА ГУРСА}

Налагая условие

$$
n^{\uparrow}=\kappa,
$$

или, что эквивалентно, $\mathbf{H}^{\uparrow}=\mathbf{K}$, можно преобразовать уравнения (10) и (22), которые эквивалентны при этом соответственно уравнениям (29) и (30), к виду, в котором естественно называть их дискретными уравнениями типа Гурса. Инвариантные характеристики дискретных уравнений типа Гурса имеют вид

$$
\left(n_{(2)}^{\uparrow}\right)^{2}=\kappa^{\uparrow} \kappa_{(12)}^{\uparrow} \diamond \frac{1}{1+\kappa^{\uparrow}}, \quad\left(\kappa_{(1)}\right)^{2}=n n_{(12)} \diamond \frac{1}{1+n} .
$$

В терминах функций, определенных в (28), получим [8]

$$
\diamond \frac{\mathcal{P}}{\mathcal{Q}}=\frac{\mathcal{M}_{(2)}}{\mathcal{M}_{(1)}} .
$$

Вводя теперь функцию $\tau$,

$$
\frac{\tau_{(12)} \tau}{\tau_{(1)} \tau_{(2)}}=\mathcal{M}=1-\mathcal{P} \mathcal{Q}
$$


и ввиду того, что уравнения (27) обладают симметрией

$$
(\mathcal{X}, \mathcal{Y}, \mathcal{P}, \mathcal{Q}) \rightarrow\left(\mathcal{X} V\left(m_{2}\right), \mathcal{Y} U\left(m_{1}\right), \mathcal{P} \frac{U\left(m_{1}\right)}{V\left(m_{2}\right)}, \mathcal{Q} \frac{V\left(m_{2}\right)}{U\left(m_{1}\right)}\right)
$$

без потери общности можно положить $\mathcal{P} \tau_{(1)}=\mathcal{Q} \tau_{(2)}$. Вводя функции

$$
x:=\mathcal{X} \sqrt{\frac{\tau}{\tau_{(2)}}}, \quad y:=\mathcal{Y} \sqrt{\frac{\tau}{\tau_{(1)}}},
$$

получим [17]

$$
x_{(1)}=\frac{1}{\sqrt{\mathcal{M}}}\left(x+\mathcal{P} \sqrt{\frac{\tau_{(1)}}{\tau_{(2)}}} y\right), \quad y_{(2)}=\frac{1}{\sqrt{\mathcal{M}}}\left(y+\mathcal{Q} \sqrt{\frac{\tau_{(2)}}{\tau_{(1)}}} x\right) .
$$

Если функции $x$ и $y$ удовлетворяют этой системе уравнений, то существует функция $\theta$ такая, что

$$
x^{2}=\Delta_{(2)} \theta, \quad y^{2}=\Delta_{(1)} \theta .
$$

Также имеем

$$
1-\mathcal{M}=\mathcal{P}^{2} \frac{\tau_{(1)}}{\tau_{(2)}}=\mathcal{Q}^{2} \frac{\tau_{(2)}}{\tau_{(1)}}
$$

а потому уравнения (55), возведенные в квадрат (дискретные аналоги уравнения (7)), принимают вид

$$
\Delta_{(1)} \Delta_{(2)} \theta=\frac{1-\mathcal{M}}{\mathcal{M}}\left(\Delta_{(1)} \theta+\Delta_{(2)} \theta\right) \pm 2 \sqrt{\frac{1-\mathcal{M}}{\mathcal{M}^{2}}} \sqrt{\Delta_{(1)} \theta} \sqrt{\Delta_{(2)} \theta}
$$

где знак \pm возникает в результате извлечения квадратного корня из выражений (56). Будем называть уравнение (57) нелинейной версией дискретного уравнения Гурса.

В случае редукции Гурса, в полной аналогии с непрерывным случаем, лестничная диаграмма перекручивается и складывается в диаграмму

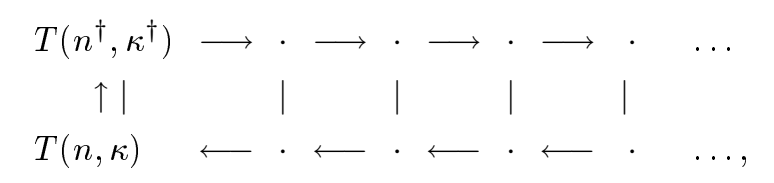

поскольку вследствие формул $(24),(40)$ и (50) получается, что $\left(n^{\uparrow \dagger}, \kappa^{\uparrow \dagger}\right)=\left(\kappa_{(1)}^{\uparrow}, n_{(2)}^{\uparrow}\right)=$ $\left(n_{(2)}, \kappa_{(2)}\right)$ и, следовательно, $\left(n^{\dagger}, \kappa^{\dagger}\right)=\left(n_{(1)}^{\uparrow}, \kappa_{(1)}^{\uparrow}\right)$, т.е.

$$
T\left(n^{\uparrow \dagger}, \kappa^{\uparrow \dagger}\right)=T(n, \kappa), \quad T\left(n^{\dagger}, \kappa^{\dagger}\right)=T\left(n^{\uparrow}, \kappa^{\uparrow}\right) .
$$

\section{9. ЗАКЛЮЧЕНИЕ}

В работе показано, что в дискретном случае уравнение Мутара расщепляется в дискретное уравнение Мутара и в уравнение, сопряженное дискретному уравнению Мутара. Эта факторизация сушественна как для физически важных [14], [18], так и для геометрически значимых [16], [19] систем.

Благодарности. Автор благодарен организаторам конференции "NEEDS 2001" за поддержку. Работа была частично поддержана KBN (грант № 2 P03B 126 22). 


\section{Список литературы}

[1] H. Jonas. Sitzungsber. Berlin. 1915. V. 14. P. 96.

[2] L. P. Eisenhart. Transformation of surfaces. Princeton: Princeton Univ. Press, 1923.

[3] В. Е. Захаров, С. В. Манаков. Функц. анализ и его прилож. 1985. Т. 19. № 2. С. 11.

[4] L. V. Bogdanov, B. G. Konopelchenko. J. Phys. A. 1995. V. 28. P. L173; M. Mañas, A. Doliwa, P. M. Santini. Phys. Lett. A. 1997. V. 232. P. 99.

[5] A. Doliwa, P. M. Santini, M. Mañas. J. Math. Phys. 2000. V. 41. P. 944.

[6] J. J. C. Nimmo, W. K. Schief. Proc. R. Soc. London A. 1997. V. 453. P. 255.

[7] J. Cieslinski, A. Doliwa, P. M. Santini. Phys. Lett. A. 1997. V. 235. P. 480; B. G. Konopelchenko, W.K. Schief. Proc. R. Soc. London A. 1998. V. 454. P. 3075; A. Doliwa, S. V. Manakov, P. M. Santini. Commun. Math. Phys. 1998. V. 196. P. 1.

[8] A. Doliwa, P. M. Santini. J. Geom. Phys. 2000. V. 36. P. 60.

[9] A. Doliwa. J. Geom. Phys. 1999. V. 30. P. 169.

[10] C. Athorne. Inverse Problems. 1993. V. 9. P. 217.

[11] E. Goursat. Bull. Soc. Math. France. 1900. V. 28. Р. 1; E. И. Ганжса. ТМФ. 2000. Т. 122. № 1. C. 50.

[12] Th.-F. Moutard. J. Ec. Pol. 1878. V. 45. P. 1.

[13] A. Doliwa. Phys. Lett. A. 1997. V. 234. P. 187; B. Э. Адлер, С. Я. Стариев. ТМФ. 1999. Т. 121. № 2. С. 271; И. А. Дьнников, С. П. Новиков. УМН. 1997. Т. 52. № 6. С. 157.

[14] M. Nieszporski, A. Doliwa, P. M. Santini. The integrable discretization of the Bianchi-Ernst system. nlin.SI/0104065; A. Doliwa, M. Nieszporski, P. M. Santini. J. Phys. A. 2001. V. 34. P. 10423 .

[15] A. Doliwa. Lattice geometry of the Hirota equation. In: SIDE III-Symmetries and Integrability of Difference Equations. Proc. of 3rd Conf. (Sabandia, Italy, May, 1998). CRM Proc. Lect. Notes. V. 25. Eds. D. Levi et al. Providence, RI: Amer. Math. Soc., 2000. P. 93.

[16] A. Doliwa. Geometric discretization of the Koenigs nets. Submitted to Geom. Dedicata.

[17] W. K. Schief. On the unification of classical and novel integrable surfaces: II. Difference geometry. nlin.SI/0104037.

[18] W. K. Schief. Stud. Appl. Math. 2001. V. 106. P. 85.

[19] M. Nieszporski. J. Geom. Phys. 2002. V. 40. P. 259. 